\title{
Advocacy Update: Is Your Practice Equipped to Handle Looming Changes in Dermatopathology?
}

\author{
Alina G. Bridges, DO; Alexandra Flamm, MD; Daniel M. Siegel, MD, MS
}

\section{PRACTICE POINTS}

- A proposed 2022 fee schedule negatively impacting dermatopathology practices has been published by the Centers for Medicare \& Medicaid Services (CMS) in July 2021.

- New pathology consultation codes with new payment rates proposed by CMS can be used starting January 1, 2022.

- The 21st Century Cures Act Final Rule has information blocking provisions.
In July 2021, the Centers for Medicare \& Medicaid Services (CMS) published a proposed fee schedule that will negatively impact practices that perform dermatopathology services. In this article, we review the proposed new and improved codes and values for pathology consultation codes as well as new payment rates. We also discuss the proposed 2022 quality payment program (QPP), and we provide an update on the 21 st Century Cures Act (Cures Act) and its impact on dermatopathology reports.

Cutis. 2021;108:267-270.

7 he proposed 2022 Medicare physician fee schedule and quality payment program (QPP) regulations were released on July 13, 2021. ${ }^{1}$ Final regulations are expected to be released on or around November 1, 2021, but they may be delayed. Multiple national medical organizations, including the College of American Pathologists (CAP), the American Society of Dermatopathology, the American Academy of Dermatology Association (AADA), and the American Medical Association (AMA) Physicians'Grassroots Network all work together to engage with the Centers for Medicare \& Medicaid Services (CMS) to influence these regulations. Stated advocacy priorities include protecting the value of dermatopathology services, mobilizing dermatopathologists for political action, ensuring dermatopathologists can participate in new payment models, strengthening the profession with advocacy on a state level, and conducting socioeconomic research. Is your practice aware and prepared to handle the changes coming in 2022?

\section{Medicare Cuts}

The recent revisions and revaluations of the outpatient evaluation and management $(\mathrm{E} / \mathrm{M}) \operatorname{codes}^{2}$ resulted in a considerable redistribution of Medicare dollars in 2021, negatively impacting dermatopathologists and other specialties and services due to budget neutrality required by law (Figure). Important steps were taken to mitigate the 2021 Medicare cuts for all non-office-based dermatopathology services (eg, pathology, surgical services, emergency department). 1,3 Direct engagement by the CAP, American Society of Dermatopathology, and AADA, along with the AMA Physicians' Grassroots Network resulted in legislative action on December 27, 2020, which directed Medicare to make a $3.75 \%$ positive adjustment to the 2021 physician payments. Additionally, the CMS updated the 2021 physician conversion factor to $\$ 34.8931$, a $3.3 \%$ reduction from the 2020 conversion factor rather

\footnotetext{
Dr. Bridges is from Richfield Laboratory of Dermatopathology, Dermpath Diagnostics, Cincinnati, Ohio. Dr. Flamm is from the Department of Dermatology, Penn State Hershey Medical Center, Pennsylvania. Dr. Siegel is from the Department of Dermatology, SUNY Downstate Medical Center, Brooklyn, New York.

The authors report no conflict of interest.

Correspondence: Alina G. Bridges, DO, Richfield Laboratory of Dermatopathology, Dermpath Diagnostics, 9844 Redhill Dr, Cincinnati, OH 45242 (alinagbridges@gmail.com).

doi:10.12788/cutis.0384
} 


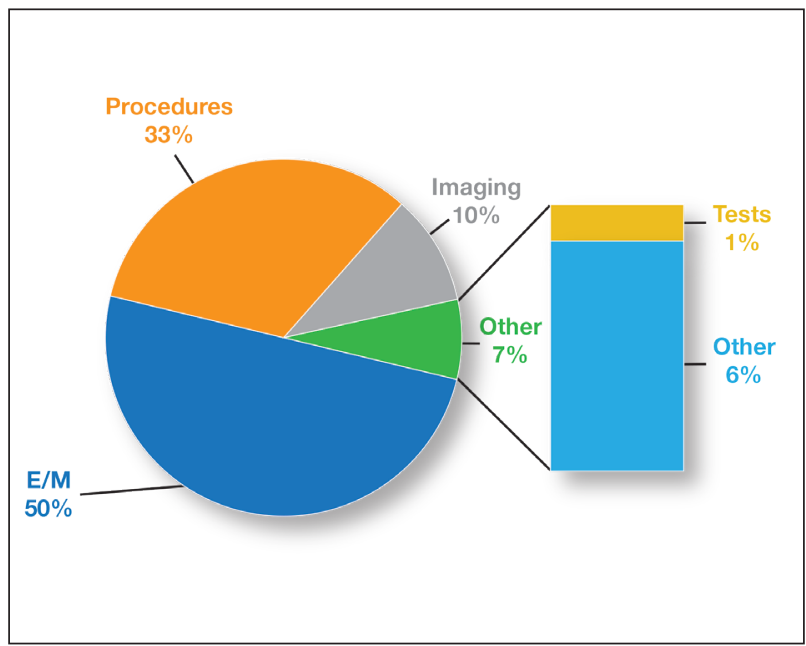

Medicare physician spending by type of service. E/M indicates evaluation and management. than $\$ 32.41$, or a $10.20 \%$ decrease. The $2 \%$ payment adjustment (sequestration) through December 21, 2021, also was suspended, and Congress and the Biden administration mandated delayed implementation of the inherent complexity add-on code for E/M services (G2211) until $2024.1,3$

\section{Threat of Medicare Cuts in 2022}

Based on dermatopathology utilization data, the overall impact on reimbursement for 2022 represents an approximately 5\% decrease from 2021 dermatopathology payments (Table 1). ${ }^{1,4}$ This represents a $3.75 \%$ cut from revaluation of $\mathrm{E} / \mathrm{M}$ services, and a $1 \%$ cut due to changes in practice expense pricing. The estimated change in reimbursement for independent laboratories is a $6 \%$ decrease. Advocacy groups have been working to mitigate the 2022 cuts by engaging with Congress and urging them to act before these changes go into effect next year. Keep in mind that approximately half of all pathology

TABLE 1. Proposed Reimbursement for Top Pathology Services in 2022

\begin{tabular}{|c|c|c|c|c|c|}
\hline CPT code & Description & Modifier $^{a}$ & 2021 Payment, \$ & $\begin{array}{l}\text { Proposed } 2022 \\
\text { payment, } \$\end{array}$ & $\begin{array}{l}\text { Change in } \\
\text { reimbursement, \% }\end{array}$ \\
\hline \multirow[t]{3}{*}{88305} & $\begin{array}{l}\text { Tissue examination by } \\
\text { a pathologist }\end{array}$ & -26 & 37.68 & 35.94 & -4.6 \\
\hline & & & 71.53 & 69.86 & -2.3 \\
\hline & & & 33.85 & 33.92 & 0.2 \\
\hline \multirow[t]{3}{*}{88312} & $\begin{array}{l}\text { Special stains, } \\
\text { microoganisms }\end{array}$ & & 26.87 & 25.52 & -5.0 \\
\hline & & None & 113.05 & 107.14 & -5.2 \\
\hline & & $\mathrm{TC}$ & 86.19 & 81.61 & -5.3 \\
\hline \multirow[t]{3}{*}{88313} & $\begin{array}{l}\text { Special stains, } \\
\text { nonmicroorganisms }\end{array}$ & -26 & 12.21 & 11.75 & -3.8 \\
\hline & & None & 81.65 & 78.92 & -3.3 \\
\hline & & $\mathrm{TC}$ & 69.44 & 67.17 & -3.3 \\
\hline \multirow[t]{3}{*}{88341} & $\begin{array}{l}\text { Immunohistochemistry/ } \\
\text { immunocytochemistry, each }\end{array}$ & -26 & 28.61 & 27.20 & -4.9 \\
\hline & additional antibody stain & None & 93.86 & 80.60 & -14.1 \\
\hline & & $\mathrm{TC}$ & 65.25 & 53.40 & -18.2 \\
\hline \multirow[t]{3}{*}{88342} & $\begin{array}{l}\text { Immunohistochemistry/ } \\
\text { immunocytochemistry, initial }\end{array}$ & -26 & 35.24 & 33.58 & -4.7 \\
\hline & single antibody stain & None & 106.08 & 95.38 & -10.1 \\
\hline & & $\mathrm{TC}$ & 70.83 & 61.80 & -12.7 \\
\hline
\end{tabular}

Abbreviation: CPT, Current Procedural Terminology.

aModifier -26 indicates professional component only, with the technical component (modifier TC) billed separately. 
Current Procedural Terminology (CPT) codes have been targeted for evaluation by the CMS since 2006. ${ }^{1,4}$

\section{Coding for Clinical Pathology Consultation Services}

The current clinical pathology consultation services (CPT codes 80500 and 80502) previously were identified as potentially misvalued for review by the AMA Relative Value Scale Update Committee's (RUC's) relativity assessment workgroup. ${ }^{4}$ Consequently, the CAP worked with the AMA's CPT Editorial Panel to delete codes 80500 and 80502, as well as to modernize and create the 4 new clinical pathology consultation codes: 80XX0, 80XX1, $80 X X 2$, and $80 X X 3$. Then the CAP worked with the RUC to develop physician work and practice expense values for the new clinical pathology consultation codes. Once the fee schedule is finalized, pathologists can begin using the new codes to bill these services in 2022 (Table 2). ${ }^{4}$

According to CPT, clinical pathology consultation services may be reported when the following criteria have been met: (1) the pathologist renders a clinical pathology consultation at the request of a physician or qualified health care professional at the same or another institution; (2) the pathology clinical consultation request relating to pathology and laboratory findings or other relevant clinical or diagnostic information requiring additional medical interpretative judgment is made; and (3) these codes are not reported in conjunction with codes 88321,88323 , and $88325 .{ }^{4}$

\section{Proposed 2022 Medicare QPP Requirements}

On July 13, 2021, the CMS also published its proposed 2022 QPP proposals that will take effect next year. ${ }^{4}$ According to the proposed regulation, nearly all dermatopathologists will be required to participate in Medicare's QPP, either through advanced alternative payment models (APMs) or the Merit-based Incentive Payment System (MIPS). The CAP has long advocated for reducing MIPS reporting burdens for dermatopathologists. In this regulation, the CMS is proposing key program changes that move the program forward but also introduce additional complexities; for example, the CMS will move forward with a new participation pathway called MIPS Value Pathways (MVPs). The CMS proposed 7 specific MVPs that align with certain clinical topics; however, it will not implement these MVPs until the 2023 MIPS performance period.

In 2022, dermatopathologists who are eligible for MIPS will have to take action to avoid penalties that reduce future Medicare Part B payments for their services. Performance in MIPS in 2022 affects Medicare Part B payments in 2024 by an increase of $9 \%$ to a decrease of $9 \%$.

In its proposed 2022 QPP regulations, the CMS proposed an increase of the performance threshold from 60 MIPS points to 75 MIPS points. It also proposed an increase of the exceptional Performance Threshold from 85 MIPS points to 89 MIPS points.

The CMS also proposed notable scoring changes for quality measures, including removing the 3-point floor for measures that can be scored against a benchmark. These measures would receive 1 to 10 points. Measures without a benchmark or that do not meet case requirements would earn 0 points, with an exception for small practices. The CMS also proposed removing bonus points for reporting additional outcomes and

TABLE 2. Pathology Clinical Consultation Services

\begin{tabular}{|c|c|c|c|}
\hline CPT code & Description & $\begin{array}{l}\text { RUC recommended } \\
\text { wRVU }\end{array}$ & $\begin{array}{l}2022 \text { proposed } \\
\text { wRVU }\end{array}$ \\
\hline $80 \times \times 0$ & $\begin{array}{l}\text { Pathology clinical consultation for a clinical problem with } \\
\text { limited review of the patient's history and medical records and } \\
\text { straightforward decision-making; when using time for code } \\
\text { selection, 5-20 min of total time is spent on the date of consultation }\end{array}$ & 0.50 & 0.43 \\
\hline $80 \times \times 1$ & $\begin{array}{l}\text { Pathology clinical consultation for a moderately complex clinical } \\
\text { problem with review of the patient's history and medical records } \\
\text { and moderate decision-making; when using time for code selection, } \\
21-40 \text { min of total time is spent on the date of consultation }\end{array}$ & 0.91 & 0.91 \\
\hline $80 \times \times 2$ & $\begin{array}{l}\text { Pathology clinical consultation for a highly complex clinical problem } \\
\text { with comprehensive review of the patient's history and medical } \\
\text { records and high level of decision-making; when using time for } \\
\text { code selection, } 41-60 \text { min of total time is spent on the date } \\
\text { of consultation }\end{array}$ & 1.80 & 1.71 \\
\hline $80 \times \times 3$ & $\begin{array}{l}\text { Pathology clinical consultation, prolonged service, each additional } \\
30 \text { min (list separately in addition to code for primary procedure) }\end{array}$ & 0.80 & 0.80 \\
\hline
\end{tabular}

Abbreviations: CPT, Current Procedural Terminology; RUC, Relative Value Scale Update Committee; wRVU, work relative value unit. 
high-priority measures beyond the 1 that is required, as well as establishing a 5-point floor for the first 2 performance periods for new measures, which is in line with the CAP's advocacy.

The Pathology Specialty Measure Set will remain the same as the 2021 set containing 6 quality measures, including the AADA-stewarded quality measure \#440 (skin cancer: biopsy reporting time-pathologist to clinician). Although the CAP recognizes the importance of prompt turnaround of biopsy reports, it also is working with the CMS and the AADA to mitigate the operational challenges dermatopathologists encounter when using this measure.

Due to advocacy from the CAP, the CMS included a CAP-proposed improvement activity on implementation of a laboratory preparedness plan to support continued or expanded patient care during the COVID-19 pandemic or another public health emergency. This plan should address how the laboratory would maintain or expand access to improve beneficiary health outcomes and reduce health care disparities.

The CAP has actively worked with the CMS to demonstrate the need for more appropriate and alternative measures and improvement activities so that pathologists can more fully participate in MIPS.

Alternative Payment Models-For those dermatopathologists who practice in an APM, the proposed 2022 QPP makes minimal changes to the advanced APM track while adding transition time for accountable care organizations in the Medicare Shared Savings Program to report on certain quality measures and increasing flexibility related to the program's quality performance standard.

\section{Cures Act 2021: To Do No Harm}

The 21st Century Cures Act (Cures Act) was signed into federal law in 2016. The Office of the National Coordinator for Health Information Technology (ONC) laid the groundwork for patients to have easier access to and control of their health information. ${ }^{5}$ The ONC's final rule, which went into effect on April 5, 2021, requires that all providers make their office notes, laboratory results, and other diagnostic reports (including dermatopathology reports) available to patients as soon as the physician's office receives an electronic copy. Penalty for noncompliance has not been determined.

There are information-blocking exceptions, but delaying access to a patient's report so that a provider can review the result before the patient receives it is not considered an exception. ${ }^{6}$ The exceptions are situational and must be evaluated by the referring clinician or their employer. Documentation of the exception is critical. The specific facts and circumstances associated with your decision to use an exception will be important to include in your documentation. Information blocking necessary to prevent "harm" to a patient or another person requires a reasonable belief that the practice will substantially reduce the risk of harm. ${ }^{6}$

The AMA passed a resolution in June 2021 calling for changes to this rule to allow for a delay of pathology results, advocating to the Office for Civil Rights to revise the harm exception to include psychological distress. ${ }^{6}$ In August 2021, the AADA met with senior officials at the ONC also asking to revise its definition of harm, sharing examples of emotional strain that resulted from receiving results without clinical context. ${ }^{7}$ California enacted a law requiring a delay before a patient receives the result of a malignant diagnosis, giving the clinician time to contact the patient before they see their report. ${ }^{8}$

The Cures Act requirements are about patients accessing their health care information. Always consider what is best for the patient and ensure that your policies and procedures reflect this. ${ }^{5}$

\section{Final Thoughts}

It is important to learn and support advocacy priorities and efforts and to join forces to protect your practice. Physician advocacy is no longer an elective pursuit. We need to be involved and engaged through our medical societies to help patients, communities, and ourselves.

\section{REFERENCES}

1. Centers for Medicare \& Medicaid Services. Calendar Year (CY) 2022 Medicare Physician Fee Schedule Proposed Rule. Published July 13, 2021. Accessed October 22, 2021. https://www.cms.gov/newsroom /fact-sheets/calendar-year-cy-2022-medicare-physician-fee-schedule -proposed-rule

2. Healthcare spending and the Medicare program. Medicare Payment Advisory Commission; July 2020. Accessed October 25, 2021. http://www.medpac.gov/docs/default-source/data-book/july2020 _databook_entirereport_sec.pdf

3. Frieden J. 2021 Medicare fee schedule includes $10.2 \%$ cut in conversion factor. MedPage Today website. Published December 2, 2020. Accessed October 22, 2021. https://www.medpagetoday.com /practicemanagement/reimbursement/89970

4. Advocacy. College of American Pathologists website. Accessed October 13, 2021. https://www.cap.org/advocacy

5. ONC's Cures Act Final Rule. The Office of the National Coordinator for Health Information Technology website. Accessed October 13, 2021. https://www.healthit.gov/curesrule/

6. Nelson H. Delegates call AMA to advocate for provider infoblocking flexibility. Published June 18, 2021. Accessed October 13, 2021. https://ehrintelligence.com/news/delegates-call-ama-to-advocate -for-provider-info-blocking-flexibility

7. Rosamilia LL. Immediate Pathology report release to patientsis the $21^{\text {st }}$ Century Cures Act worse than the disease? American Academy of Dermatology website. Published August 25, 2021. Accessed October 22, 2021. https://www.aad.org/dw/dw-insights-and -inquiries/archive/2021/cures-act-immediate-pathology -report-release-to-patients

8. Purington K, Alfreds ST, Pritts J, et al; The National Academy for State Health Policy. Electronic release of clinical laboratory results: a review of state and federal policy. Published January 2010. Accessed October 13, 2021. https://www.nashp.org/wp-content/uploads/2010/02 /ElectronicLabResultsExchangePolicy.pdf 